\title{
How to follow-up patients with hereditary haemorrhagic telangiectasia and suspected pulmonary arteriovenous malformations
}

\author{
Thierry Chinet ${ }^{1,2}$
}

Affiliations: ${ }^{1}$ Université de Versailles Saint-Quentin-en-Yvelines UFR médicale Paris lle de France Ouest, Guyancourt, France. ${ }^{2}$ Assistance Publique-Hôpitaux de Paris, Service de Pneumologie et Oncologie Thoracique, Consultation Pluridisciplinaire Maladie de Rendu-Osler, Boulogne-Billancourt, France.

Correspondence: Thierry Chinet, Hôpital Ambroise Paré, Service de Pneumologie et Oncologie Thoracique, Consultation Pluridisciplinaire Maladie de Rendu-Osler, 9 avenue Charles de Gaulle, Boulogne, 92100, France. E-mail: thierry.chinetQaphp.fr

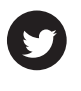

@ERSpublications

Contrast echocardiography is useful for the follow-up of HHT patients with pulmonary arteriovenous malformations http://ow.ly/YNql5

Pulmonary arteriovenous malformations (PAVMs) are abnormal vascular structures that connect one or several pulmonary arteries to one or several pulmonary veins without interposition of a capillary bed, resulting in a right-to-left shunt (RLS) [1]. Over $80 \%$ of PAVMs are associated with the genetic disease hereditary haemorrhagic telangiectasia (HHT). The vascular malformations predispose patients to severe complications due to paradoxical systemic emboli of thrombotic or septic origin, such as stroke and brain abscess [2-5]. The treatment of choice for PAVMs is transcatheter embolotherapy, although concerns are emerging regarding the long-term consequences of this procedure, especially in children [6].

Because of the risk of severe complications, international guidelines recommend screening patients with HHT for the presence of PAVMs at the time of initial clinical evaluation and also after puberty, after pregnancy, within 5 years preceding a planned pregnancy, and otherwise every 5-10 years [7]. Transthoracic contrast echocardiography (TTCE) is the first-line screening technique for the detection of PAVMs in HHT [7]. In expert hands, it has excellent sensitivity and negative predictive value for the presence of PAVMs in children and adults. Several grading scales are used in different HHT centres to quantify the pulmonary RLS size [6-8]. Microbubbles of air in agitated saline may expose patients with RLS to the risk of cerebral air emboli inducing migraine, blurred vision, numbness and paraesthesia, but these symptoms resolve quickly without residual side effects $[9,10]$. To minimise this risk, many groups perform a chest radiograph prior to TTCE to detect large-size PAVMs, which are likely to carry the highest risk of complications from air bubbles. TTCE is therefore a safe diagnostic tool and its benefits undoubtedly outweigh its potential minor risks.

One must bear in mind that TTCE does not detect PAVMs but rather pulmonary RLS. Chest computed tomography (CT) is considered the gold standard diagnostic tool for PAVMs as it enables visualisation of the vascular malformations (except microscopic ones) and provides essential information on their characteristics (shape, location, size of the feeding arteries, size of the aneurismal sac, etc.). Recent studies have reported that, in patients with HHT, the grade of pulmonary RLS on TTCE predicts the size of PAVMs on chest CT and the feasibility of subsequent transcatheter embolotherapy [7]. The absence of, or a small, pulmonary RLS (i.e. a low grade on TTCE) is associated with no risk of neurological complications and with PAVMs too small for embolisation $[5,7]$.

Received: Feb 152016 | Accepted after revision: Feb 202016

Conflict of interest: None declared.

Copyright OERS 2016 
The natural history of PAVMs is not well known. There is some evidence that PAVMs may grow not only in children but also in adults, spontaneously or after transcatheter embolotherapy of other larger PAVMs in the same patient [11]. However, in adults, it is not clear whether it is only growth of previously small PAVMs that occurs, or whether de novo PAVMs may also appear. This point is important, because if truly new fistulas can develop in adult patients, a negative initial screening, even using a highly sensitive test, cannot rule out the development of PAVMs in the future, necessitating repeated screenings during the lifetime. The possible growth of PAVMs during adulthood implies repeated follow-up evaluations to detect when the PAVMs become large enough to be embolised (based on a diameter of the feeding arteries of $>2-3 \mathrm{~mm}$ ). However, the precise method and the appropriate screening interval are not agreed upon. Practices differ from one centre to another.

In this issue of the European Respiratory Journal, VORSELAARs et al. [12] retrospectively reviewed change in RLS evaluated using TTCE at 5 years in 200 HHT patients. At the initial screening, all patients but one had no RLS or RLS but a nontreatable PAVM. This study found that an increase in pulmonary RLS grade occurred in $18 \%$ of patients (both with and without RLS at screening), while a decrease was observed in 7.5\%. 55 patients had an RLS grade $\geqslant 2$ on follow-up and had a chest CT. 14 of these patients underwent embolotherapy.

This work contributes noteworthy findings and clues for future investigation. First, it confirms that over a period of 5 years, PAVMs may grow in a significant percentage of HHT patients who did not have previous embolotherapy. When chest CT was repeated 5 years after screening in patients with RLS grade $\geqslant 2$, the diameter of the feeding artery of the largest PAVM exceeded $2.1 \mathrm{~mm}$ in 15 patients versus in seven at baseline. This resulted in embolotherapy in 14 patients, although it is not clear whether this procedure was performed only because the PAVMs grew or because the authors' experience with embolotherapy had improved in recent years, with improved technical ability to occlude smaller PAVMs (i.e. with a feeding artery of 2-3 mm). This study therefore provides further justification for the recommendation to follow-up HHT patients who have nontreatable PAVMs.

Secondly, this study indicates that RLS may appear during follow-up in HHT adult patients with no pulmonary RLS at the initial screening. Although a grade 1 RLS in a patient with a previously grade 0 RLS is difficult to interpret, it is certainly unwise not to rescreen patients who were initially negative, especially because 10 patients with a grade 1 RLS at screening had a grade 2 RLS 5 years later.

Thirdly, this study found that the increase in RLS is never more than one grade over 5 years. Therefore, follow-up in patients with no pulmonary RLS at screening could theoretically be safely deferred to more than 5 years, although this remains to be confirmed in other cohorts.

Fourthly, this study promotes the use of TTCE rather than chest CT in the screening and follow-up of HHT patients with low-grade RLS. Patients with HHT, including children and young adults, are exposed to a significant cumulative radiation dose from diagnostic and therapeutic interventions, which are associated with harmful effects in the long term [13]. Recently, Mathew et al. [14] studied cancer risk in 680211 people exposed to CT scans in childhood or adolescence. Overall, the cancer incidence during a mean follow-up of 9.5 years was $24 \%$ greater in exposed than in non-exposed people. Chest CT to detect PAVMs can safely be avoided in patients with an absence of, or grade 1, RLS on TTCE $[8,10,15]$.

There are still numerous areas of uncertainty. TTCE lacks specificity and has a low positive predictive value for the presence of PAVMs. In this study, 23 HHT patients with a grade 2 or 3 RLS had no PAVM on chest CT at the initial screening, which is consistent with the finding that $6-28 \%$ of the normal population has a positive TTCE [15-17]. This can reflect true false positive results or microscopic PAVMs that are too small to be detected with chest CT. In any case, an increase in RLS could be due to the development of PAVMs or to other unknown and possibly unrelated factors. A chest CT is clearly required at least in the case of a grade $\geqslant 2$ RLS. The reproducibility of TTCE in a particular patient is not clear, meaning that comparisons between screening and follow-up results must be treated with caution. Surprisingly, a decrease in pulmonary RLS grade was seen in $7.5 \%$ of patients. The authors evaluated the reproducibility of TTCE per patient by performing two separate contrast injections in 30 patients, and found good agreement between the assigned grades. However, this evaluation was conducted during the same procedure, in a small number of patients, in an experienced centre, and needs to be further defined in a larger cohort of patients and in various centres.

This was a single-centre study, performed by a team with a high level of expertise in TTCE and PAVMs. As outlined by the authors, a prospective multicentre validation study is required to confirm these results. TTCE has no value for the follow-up of patients with known untreated macroscopic PAVMs and a grade $\geqslant 2$ RLS. However, the optimal method for the follow-up of patients with macroscopic PAVMs and a grade 1 RLS is still uncertain. In this study, out of eight of these patients, five progressed from grade 1 to grade 2 
RLS and therefore had a chest CT (three underwent embolotherapy); three remained at grade 1 RLS and did not have a chest CT. This group of patients needs to be investigated further. Finally, TTCE cannot be used to follow patients with PAVMs who have undergone previous embolisation, because it remains positive in almost all of them, and chest CT is required to evaluate the late complications of embolisation such as reperfusion $[11,18-20]$.

At this point, what should the follow-up strategy be for clinicians in charge of HHT patients? We obviously need to adapt our protocols to particular groups of patients according to the results of the initial screening procedure. Patients with no RLS can safely be followed-up using TTCE: a chest CT is not necessary to screen for PAVMs in these patients. Whether an interval of more than 5 years before the next evaluation of these patients is safe requires more data. Patients with a grade 2 or 3 RLS on initial screening or during follow-up should definitely undergo a chest CT. More research is necessary for patients with a grade 1 RLS. Some centres will perform a chest CT, while others will not, until further data are available.

The optimal interval between evaluations for patients with RLS on TTCE, or with PAVMs on chest CT, remains to be determined and is very likely to differ according to the patient's age, the grade of RLS, the characteristics of PAVMs on CT, previous embolisations, etc. Again, more research is necessary.

The follow-up of patients with treated PAVMs cannot rely on TTCE [10]. International guidelines indicate that chest CT should be performed within 6-12 months after embolisation and then approximately every 3 years to detect reperfusion of treated PAVMs and growth of untreated PAVMs. However, there has been no comparative study to determine the optimal method to detect reperfusion of PAVMs and the optimal interval. Interestingly, a recent paper reported that reperfusion of occluded PAVMs could be predicted by a diameter of the draining vein of $2.5 \mathrm{~mm}$ or more on unenhanced chest CT [21]. The use of unenhanced chest CT rather than contrast-enhanced CT might limit the risk of paradoxical emboli due to venous injection of the contrast agent in these patients.

Follow-up with chest CT in patients with PAVMs is not satisfactory because, as discussed above, it exposes often young patients to the harmful effects of large amounts of radiation. New magnetic resonance imaging techniques might prove useful in the future.

\section{References}

1 Cartin-Ceba R, Swanson KL, Krowka MJ. Pulmonary arteriovenous malformations. Chest 2013; 144: 1033-1044.

2 Kjeldsen AD, Oxhøj H, Andersen PE, et al. Prevalence of pulmonary arteriovenous malformations (PAVM) and occurrence of neurological symptoms in patients with hereditary haemorrhagic telangiectasia (HHT). J Intern Med 2000; 248: 255-262.

3 Moussouttas M, Fayad P, Rosenblatt M, et al. Pulmonary arteriovenous malformations: cerebral ischemia and neurologic manifestations. Neurology 2000; 55: 959-964.

4 Shovlin CL, Jackson JE, Bamford KB, et al. Primary determinants of ischaemic stroke/brain abscess risks are independent of severity of pulmonary arteriovenous malformations in hereditary haemorrhagic telangiectasia. Thorax 2008; 63: 259-266.

5 Velthuis S, Buscarini E, van Gent MW, et al. Grade of pulmonary right-to-left shunt on contrast echocardiography and cerebral complications: a striking association. Chest 2013; 144: 542-548.

6 Karam C, Sellier J, Mansencal N, et al. Reliability of contrast echocardiography to rule out pulmonary arteriovenous malformations and avoid CT irradiation in pediatric patients with hereditary hemorrhagic telangiectasia. Echocardiogaphy 2015; 32: 42-48.

7 Velthuis S, Buscarini E, Mager JJ, et al. Predicting the size of pulmonary arteriovenous malformations on chest computed tomography: a role for transthoracic contrast echocardiography. Eur Respir J 2014; 44: 150-159.

8 Parra JA, Bueno J, Zarauza J, et al. Graded contrast echocardiography in pulmonary arteriovenous malformations. Eur Respir J 2010; 35: 1279-1285.

9 Arthur H, Geisthoff U, Gossage JR, et al. Executive summary of the 11th HHT international scientific conference. Angiogenesis 2015; 18: 511-524.

10 Faughnan ME, Palda VA, Garcia-Tsao G, et al. International guidelines for the diagnosis and management of hereditary haemorrhagic telangiectasia. J Med Genet 2011; 48: 73-87.

11 Pollak JS, Saluja S, Thabet A, et al. Clinical and anatomic outcomes after embolotherapy of pulmonary arteriovenous malformations. J Vasc Interv Radiol 2006; 17: 35-45.

12 Vorselaars VMM, Velthuis S, Snijder RJ, et al. Follow-up of pulmonary right-to-left shunt in hereditary haemorrhagic telangiectasia. Eur Respir J 2016; 47: 1750-1757.

13 Hanneman K, Faughnan ME, Prabhudesai V. Cumulative radiation dose in patients with hereditary hemorrhagic telangiectasia and pulmonary arteriovenous malformations. Can Assoc Radiol J 2014; 65: 135-140.

14 Mathews JD, Forsythe AV, Brady Z, et al. Cancer risk in 680,000 people exposed to computed tomography scans in childhood or adolescence: data linkage study of 11 million Australians. BMJ 2013; 346: f2360.

15 Velthuis S, Buscarini E, Gossage JR, et al. Clinical implications of pulmonary shunting on saline contrast echocardiography. J Am Soc Echocardiogr 2015; 28: 255-263.

16 Elliott JE, Nigam SM, Laurie SS, et al. Prevalence of left heart contrast in healthy, young, asymptomatic humans at rest breathing room air. Respir Physiol Neurobiol 2013; 188: 71-78.

17 van Gent MW, Post MC, Luermans JG, et al. Screening for pulmonary arteriovenous malformations using transthoracic contrast echocardiography: a prospective study. Eur Respir J 2009; 33: 85-91.

18 Lee WL, Graham AF, Pugash RA, et al. Contrast echocardiography remains positive after treatment of pulmonary arteriovenous malformations. Chest 2003; 123: 351-358. 
19 Milic A, Chan RP, Cohen JH, et al. Reperfusion of pulmonary arteriovenous malformations after embolotherapy. $J$ Vasc Interv Radiol 2005; 16: 1675-1683.

20 Lacombe P, Lacout A, Marcy PY, et al. Diagnosis and treatment of pulmonary arteriovenous malformations in hereditary hemorrhagic telangiectasia: an overview. Diagn Interv Imaging 2013; 94: 835-848.

21 Gamondès D, Si-Mohamed S, Cottin V, et al. Vein diameter on unenhanced multidetector CT predicts reperfusion of pulmonary arteriovenous malformation after embolotherapy. Eur Radiol 2015 [in press; DOI: 10.1007/s00330-015-4090-8]. 